\title{
The Yield of Lumbosacral Spine MRI in Patients with Isolated Chronic Low Back Pain: A Cross-Sectional Study
}

This article was published in the following Dove Press journal: Orthopedic Research and Reviews

\author{
Tareq Kanaan (1D' \\ Mohammed Alisi (iD) ${ }^{2}$ \\ Yara Anasweh (iD) ${ }^{3}$ \\ Noor Yousef (iD ${ }^{2}$ \\ Qussay Al-Sabbagh (1) \\ Fadi Hadidi ${ }^{2}$ \\ Abdul Rahman Al-Shudifat \\ 'Department of Special Surgery, \\ Division of Neurosurgery, School of \\ Medicine, The University of Jordan, \\ Amman, Jordan; ${ }^{2}$ Department of Special \\ Surgery, Division of Orthopaedics, \\ School of Medicine, The University of \\ Jordan, Amman, Jordan; ${ }^{3}$ Department of \\ Anesthesia and Intensive Care, School \\ of Medicine, The University of Jordan, \\ Amman, Jordan
}

Background: The role of routine lumbosacral MRI in patients presented with isolated chronic low back pain (CLBP) is still unclear. Most patients with CLBP will show diverting degenerative changes on MRI. As it is uncertain whether surgical treatment of degenerative MRI changes results in alleviation of back pain or not, the necessity of doing a diagnostic lumbosacral MRI remains questionable. This study aimed to evaluate the yield of lumbosacral MRI among Jordanian patients presented with isolated CLBP.

Methods: We reviewed medical records of all patients who presented to neurosurgery outpatient clinic at Jordan University Hospital from December 2016 to December 2019. Only patients with a chief complaint of isolated CLBP were included. We obtained the relevant data from the computerized medical files and detailed radiological findings from their MRI reports.

Results: One hundred and sixty-seven patients (167) matched the inclusion criteria. We reported positive findings in MRI in 112 patients (67\%), but 55 patients (32.93\%) had normal MRI findings. Dehydration of intervertebral disc was the most common finding. Positive MRI findings were most evident in the middle-age group (41-60 years old). Disc protrusion finding in middle-aged females was significantly less prevalent than males $(P=0.012)$.

Conclusion: Jordanian patients presented with CLBP have similar worldwide patterns of lumbar degenerative changes. Providing that near one-third of patients with CLBP have normal MRI findings, we suggest following a streamlined protocol for imaging of patients presented with CLBP to reduce healthcare costs.

Keywords: chronic low back pain, MRI findings, degenerative changes

\section{Introduction}

Low back pain (LBP) is a very common complaint; about $67 \%$ to $84 \%$ of people in industrialized countries are affected by LBP at some point in their lives. ${ }^{1,2}$ In the United States, about $25 \%$ of adults have experienced LBP for at least one day in the past three months. In people younger than 45 years, LBP is the most common cause of limited activity and work absence, which leads to decreased productivity and a greater economic burden. ${ }^{1,3,4}$ Consequently, this will negatively affect the individual's socioeconomic level. It is approximated that $5 \%$ of patients with LBP attribute to $75 \%$ of healthcare costs. Economic losses, both direct and indirect in the United States have been calculated to be almost 90 billion dollars annually as a result of LBP.

Chronic low back pain (CLBP) is defined as low back pain persisting for 3 months and more that does not stem from a clearly defined pathology such as
Correspondence: Mohammed Alisi

School of Medicine, The University of Jordan

Queen Rania Al Abdullah St 266 Al Jubaiha,

Amman I 1942, Jordan

Tel +962790983284

Email m.elessi2007@hotmail.com
Orthopedic Research and Reviews 2020:12 139-143 
fracture, deformity, neural compression, infection, or neoplasm. ${ }^{5}$ The lifetime prevalence of LBP is $60-85 \%$. About $15 \%$ of adults will have LBP at any one time. ${ }^{3}$

Lumbar disc degeneration disease (LDDD) is a wide term used to describe the anatomical age-related changes involving the intervertebral discs and the facet joints. It is the most common cause of both acute and chronic LBP. MRI is the golden standard tool for the diagnosis of degenerative spine and spine pathologies. ${ }^{6}$ Aside from confirming the diagnosis or excluding other relevant pathologies, its role as a routine investigation in patients with isolated CLBP is unclear. Also, its effect on the line of management of patients with isolated CLBP is still a debate that surrounds its yield and value. ${ }^{5}$

We aimed in this study to evaluate the yield of lumbosacral MRI in Jordanian patients with isolated CLBP, with further analysis of age groups and gender distribution.

To our knowledge, this is the first study that comes from Jordan describing the characteristics of patients with CLBP and their radiological findings on MRI in the general population.

Few previous studies reported the prevalence and factors associated with LBP in certain occupations in Jordanians such as school teachers, nurses, and other health professionals. ${ }^{7-10}$

\section{Patients and Methods}

Our selection criteria included all patients presented with isolated CLBP of more than 3 months, without radiation to lower limbs or any neurological deficit. All patients had visited our outpatient clinics and investigated by lumbosacral MRI at Jordan University Hospital (JUH). The period of study is selected randomly, which was from December 2016 to December 2019. We excluded the patients with a history of previous back surgery, age below 18 years, and those with a known spinal or rheumatological disorders.
We collected all required data retrospectively from the outpatient computerized medical records and radiology department records. The authors certify that this research was approved by the institutional review board at Jordan University Hospital and this study complied with the Declaration of Helsinki. We obtained informed consent from all included patients to have their data to be used in this study.

Statistical analysis was done using SPSS version 20.0 (SPSS, Chicago, IL, USA). Descriptive statistics in the form of percent, mean, and standard deviation were included. Data for the middle-age group (41-60 years) for males and females were compared using the Fisher exact test, with $\mathrm{p}<0.05$ set as a level of significance.

\section{Results}

\section{Study Population}

Out of a total of 167 patients complaining of isolated CLBP, 92 (55\%) patients were males and 75 (44.91\%) patients were females. One hundred and twelve (67\%) patients were found to have positive MRI findings, 49 of them were females $(43.75 \%)$ and 63 were males $(56.25 \%)$. Age ranged from 20 to 87 years. The majority of patients with positive MRI findings were of the middle-age group (41-60 years) as shown in Table 1.

\section{MRI Findings}

The MRI findings in lumbar spine degenerative disease may include, but are not limited to, dehydrated lumbar discs, disc bulge, disc protrusion, Modic changes, facet joint problems, and spondylolisthesis. Those findings repeatedly coexisted in most patients. Our analysis showed that only 7 patients $(6.25 \%)$ had one finding, while 105 patients $(93.75 \%)$ had two or more findings.

Disc dehydration was the most common finding in both genders, it was in $95.53 \%$ of patients who have positive

Table I Age and Gender Distributions of Patients

\begin{tabular}{|l|l|l|l|}
\hline & Male & Female & Total \\
\hline $\begin{array}{l}\text { No MRI findings } \\
\text { Positive MRI findings }\end{array}$ & 29 & 26 & $55 / 167$ \\
Mean age for patients with positive MRI findings & 63 & 49 & $51.88 \pm 14.40$ \\
\hline Age subgroups for patients with positive MRI findings & Male & Female \\
\hline$<20$ years & 1 & 0 & Total \\
$20-40$ years & 20 & 12 & $1 / 167$ \\
$4 I-60$ years & 31 & 19 & $32 / 112$ \\
$>60$ years & 11 & 18 & $50 / 112$ \\
\hline
\end{tabular}


findings. The least revealing finding was spondylolisthesis $(13.39 \%)$. In the elderly-age group ( $>60$ years old), the two most common findings were disc dehydration (25.89\%) and diffuse disc bulge (24.1\%) (Table 2).

Type 2 Modic changes were the most common type seen in all patients who have Modic changes (15 of 27 patients). It was more prevalent in males than females but this was not statistically significant $(\mathrm{P}=0.569)$. We did not find any type 3 Modic changes in our study population.

None of the patients who had positive MRI findings underwent any spine surgery based on these findings.

\section{Comparison Between MRI Findings in the Middle-Age Group (4I-60 Years Old)}

Fisher exact test was used to compare MRI findings between males and females in the middle-age group population. The results showed that disc protrusion was significantly low prevalent in the female group $(\mathrm{P}=0.012)$ (Table 3).

\section{Discussion}

There is a consensus among the majority of spine-care providers that CLBP is mainly a mobility-related mechanical problem that is affected and can be exacerbated by social and psychological factors. Significant agreement on the etiology remains weak. ${ }^{1,3}$ Looking for a pathology that could explain nature and help in the management of CLBP is the main concern for researchers in this field.
Lumbosacral MRI is considered a valuable tool in illustrating degenerative spine changes, but with doubtful value regarding management. ${ }^{11}$ Although MRI has a multitude of advantages, it carries a number of limitations; its relatively high cost, ${ }^{12}$ poor patient tolerance, as well as the prolonged waiting time for an MRI appointment. At our institution, the average time for elective MRI appointment is 6 months. These limitations render its accessibility as a tool for investigation. At our institution, we routinely do lumbosacral MRI for patients with refractory CLBP aiming to find possible causes or to exclude others. Anxious patients seeking reassurance remains one of the major dilemmas in clinical practice for requesting an MRI. This in turn leads to over investigation and subsequently, a prolonged waiting time. In this study, MRI is negative in about one-third of patients $(32.93 \%)$ who presented with CLBP. Thus, implementing a streamlined protocol for imaging patients with CLBP may save the cost for the patient and healthcare system and shorten the time of waiting for patients who truly indicated for imaging.

We think that a lack of enough time for discussion of the problem with the patient is the main issue that should be addressed.

There is no strong evidence supporting the efficacy of surgical treatment of degenerative MRI changes, thus a strong recommendation has been made against treating CLBP surgically based only upon degenerative MRI changes. ${ }^{5}$ Moreover, multiple randomized clinical trials

Table 2 Distribution of MRI Findings According to Gender and Age Group

\begin{tabular}{|c|c|c|c|c|c|c|c|c|c|}
\hline \multirow[t]{2}{*}{ Finding } & \multicolumn{4}{|c|}{ Age Groups in Years/Male } & \multicolumn{5}{|c|}{ Age Groups in Years/Female } \\
\hline & $<20$ & $20-40$ & $41-60$ & $>60$ & $<20$ & $20-40$ & $4 I-60$ & $>60$ & Total \\
\hline Disc bulge & I (I.I4\%) & 14 (15.91\%) & $24(27.27 \%)$ & II (12.50\%) & 0 & 8 (09.09\%) & 14 (I5.91\%) & $16(18.18 \%)$ & 88 \\
\hline Ligament hypertrophy & 0 & $3(09.68 \%)$ & $5(16.13 \%)$ & $8(25.81 \%)$ & 0 & 0 & $3(09.68 \%)$ & $12(38.7 \mid \%)$ & 31 \\
\hline Disc dehydration & I (0.93\%) & 19 (I7.76\%) & $30(28.04 \%)$ & II (I0.28\%) & 0 & $10(09.35 \%)$ & $18(16.82 \%)$ & I8 (16.82\%) & 107 \\
\hline Disc protrusion & 0 & 4 (II.43\%) & $13(37.14 \%)$ & 2 (05.7I\%) & 0 & 4 (I I.43\%) & $5(\mid 4.29 \%)$ & 7 (20.00\%) & 35 \\
\hline Modic changes & 0 & $2(07.41 \%)$ & $8(29.63 \%)$ & $6(22.22 \%)$ & 0 & $4(|4.8| \%)$ & $5(\mid 8.52 \%)$ & $2(07.41 \%)$ & 27 \\
\hline Type I Modic & 0 & 2 & 2 & 1 & 0 & 4 & 2 & I & 12 \\
\hline Type 2 Modic & 0 & 0 & 6 & 5 & 0 & 0 & 3 & I & 15 \\
\hline Facet joint degeneration & 0 & $6(10.71 \%)$ & $12(21.43 \%)$ & $10(17.86 \%)$ & 0 & $5(08.93 \%)$ & II (19.64\%) & $12(21.43 \%)$ & 56 \\
\hline Spondylolisthesis & 0 & 0 & $3(20.00 \%)$ & $3(20.00 \%)$ & 0 & I (06.67\%) & $2(13.33 \%)$ & $6(40.00 \%)$ & 15 \\
\hline Schmorl nodules & 0 & $2(06.67 \%)$ & 7 (23.33\%) & $3(10.00 \%)$ & 0 & $3(10.00 \%)$ & $8(26.67 \%)$ & 7 (23.33\%) & 30 \\
\hline
\end{tabular}


Table 3 Comparison Between Middle-Age Group (4I-60 Years) Males and Females MRI Findings

\begin{tabular}{|l|l|l|l|}
\hline MRI Finding & Male & Female & Significance (P-value) \\
\hline Disc bulge & $24(27.27 \%)$ & $14(15.91 \%)$ & 0.053 \\
\hline Ligamentum flavum hypertrophy & $5(16.13 \%)$ & $3(09.68 \%)$ & 0.336 \\
\hline Dehydration & $30(28.04 \%)$ & $18(16.82 \%)$ & 0.126 \\
\hline Disc protrusion & $13(37.14 \%)$ & $5(14.29 \%)$ & 0.012 \\
\hline Modic changes & $8(29.63 \%)$ & $5(18.52 \%)$ & 0.234 \\
\hline Facet joint & $12(21.43 \%)$ & $11(19.64 \%)$ & 0.457 \\
\hline Spondylolisthesis & $3(20.00 \%)$ & $2(13.33 \%)$ & 0.346 \\
\hline Schmorl nodules & $7(23.33 \%)$ & $8(26.67 \%)$ & 0.497 \\
\hline
\end{tabular}

have shown that early imaging vs conservative treatment (without imaging) for patients with no red flags does not enhance patient outcomes. ${ }^{13}$ In this study, none of the patients treated surgically based on the degenerative changes on MRI.

Many studies have been examined the prevalence of lumbar spine MRI changes among different age groups. Cheung et al studied the prevalence of lumbar disc degeneration (LDD) in the Chinese population. The results showed that the prevalence of LDD in people below the age of 30 years was $40 \%$ and escalated to over $90 \%$ between the ages of 50 to $55 .{ }^{14}$ Moreover, Al-Saeed et al reported that a reduced disc signal followed by disc bulge was the most common MRI features seen in the symptomatic young Arabs. ${ }^{15}$ In comparison with these studies, our results also showed that disc degenerative changes (dehydration, bulge, protrusion) were the most common findings comprising about $(60 \%)$ of all findings mainly occurring in the middle-age group since this age group comprises a high percentage of our cohort. Based on these findings, we can say that Jordanian people of Arab descent follow the general worldwide pattern of lumbar spine degenerative changes.

A systematic review was conducted by Meucci et al to investigate the prevalence of CLBP according to age and sex. They concluded that CLBP is more prevalent in the age group (20-59 years) and more common in females. ${ }^{16}$ In our cohort, we tested gender variation in patients presented with isolated CLBP. We found that CLBP is more common in males in the overall studied group. Also, positive MRI findings are more prevalent in male patients. This may be explained by the cultural and lifestyle issues in Jordan. Interestingly, we found that disc protrusion is significantly more common in males $(\mathrm{P}=0.012)$. This finding may necessitate further follow up for this group to monitor the progress of their CLBP.

This study provides valuable basic information about the lumbar degenerative changes among the Jordanian population. Based on the findings of this study, we suggest that the management of patients with CLBP according to a clearly defined protocol will save the cost and preserve the resources of the healthcare system, especially in low-mid income countries. This protocol should include a thorough history and physical examination, starting with non-pharmacological modalities, imaging the patients who have CLBP with red flags, and activation of a multidisciplinary team for the care of those patients. The multidisciplinary should involve a pain therapy specialist, physical therapist, and psychiatrist in addition to orthopedic/neurosurgery specialists.

Limitations of this study include the retrospective design of the study as well as the relatively small sample size. The small sample size is attributed to the strict inclusion criteria. Still, we focused on specific findings in different age groups and explored gender variations in regard to these findings.

Besides, this study did not mention other MRI findings that are related to CLBP such as multifidus atrophy and signs of posterior ligaments stress/overload. Absence of routine reporting of these findings by radiologist at our institution precludes accurate description. Recently, Faur et $\mathrm{al}^{17}$ reported a low correlation between fatty atrophy of lumbar multifidus muscle and low back pain. We suggest further studies to evaluate the correlation between the degree of degenerative changes and 
fatty atrophy of multifidus muscle. Using accurate assessment tools such as Adobe Photoshop for qualitative image analysis by measuring the cross-sectional area of the pure fat component of lumbar multifidus may be helpful.

\section{Conclusion}

Lumbar degenerative changes among Jordanian patients presented with CLBP follow the worldwide pattern. Providing that near one-third of patients with CLBP have normal MRI findings, we suggest following a streamlined protocol for imaging of patients presented with CLBP to reduce healthcare costs. Lumbar spine degenerative changes should be correlated with the clinical picture of the patient and should not be the basis of surgical intervention.

\section{Disclosure}

The authors report no conflicts of interest for this work.

\section{References}

1. Fourney DR, Andersson G, Arnold PM, et al. Chronic low back pain: a heterogeneous condition with challenges for an evidence-based approach. Spine (Phila Pa 1976). 2011;36(21Suppl):S1-S9. doi:10.1097/BRS.0b013e31822f0a0d

2. Koes BW, van Tulder MW, Thomas S. Diagnosis and treatment of low back pain. BMJ. 2006;332(7555):1430-1434. doi:10.1136/ bmj.332.7555.1430

3. Krismer M, van Tulder M, Low Back Pain Group of the Bone and Joint Health Strategies for Europe Project. Strategies for prevention and management of musculoskeletal conditions. Low back pain (nonspecific). Best Pract Res Clin Rheumatol. 2007;21(1):77-91. doi:10.1016/j.berh.2006.08.004

4. Qaseem A, Wilt TJ, McLean RM, Forciea MA, Clinical Guidelines Committee of the American College of Physicians. Noninvasive treatments for acute, subacute, and chronic low back pain: a clinical practice guideline from the American College of Physicians. Ann Intern Med. 2017;166(7):514-530. doi:10.7326/M16-2367

5. Chou D, Samartzis D, Bellabarba C, et al. Degenerative magnetic resonance imaging changes in patients with chronic low back pain: a systematic review. Spine. 2011;36(21 Suppl):S43-S53. doi:10.1097/ brs.0b013e31822ef700
6. Beattie PF, Meyers SP. Magnetic resonance imaging in low back pain: general principles and clinical issues. Phys Ther. 1998;78 (7):738-753. doi:10.1093/ptj/78.7.738

7. Alghwiri A, Marchetti G. Occupational back pain among schoolteachers in Jordan: estimated prevalence and factors associated with self-reported pain and work limitations. Int J Occup Saf Ergon. 2018;24(3):341-346. doi:10.1080/10803548.2016.1247605

8. Suliman M. Prevalence of low back pain and associated factors among nurses in Jordan. Nurs Forum. 2018;53(4):425-431. doi:10.1111/nuf.12269

9. Shawashi TO, Subih MM, Al Hadid LA, Abu Adas M. Occupationalrelated back pain among Jordanian nurses: a descriptive study. Int $J$ Nurs Pract. 2015;21(Suppl 2):108-114. doi:10.1111/ijn.12168

10. Boran A, Shawaheen M, Khader Y, Amarin Z, Hill Rice V. Workrelated stress among health professionals in northern Jordan. Occup Med (Lond). 2012;62(2):145-147. doi:10.1093/occmed/kqr180

11. Chou R, Qaseem A, Owens DK, Shekelle P, Clinical Guidelines Committee of the American College of Physicians. Diagnostic imaging for low back pain: advice for high-value health care from the American College of Physicians [published correction appears in Ann Intern Med. 2012 Jan 3;156(1 Pt 1):71]. Ann Intern Med. 2011;154:181-189. doi:10.7326/0003-4819-154-3-201102010-00008

12. Millán Ortuondo E, Cabrera Zubizarreta A, Muñiz Saitua J, Sola Sarabia C, Zubia Arratibel J. Indicaciones de la resonancia magnética en la lumbalgia de adultos [Indications for magnetic resonance imaging for low back pain in adults]. Rev Calid Asist. 2014;29 (1):51-57. doi:10.1016/j.cali.2013.10.004

13. Roudsari B, Jarvik JG. Lumbar spine MRI for low back pain: indications and yield. AJR Am $J$ Roentgenol. 2010;195(3):550-559. doi:10.2214/AJR.10.4367

14. Cheung KM, Karppinen J, Chan D, et al. Prevalence and pattern of lumbar magnetic resonance imaging changes in a population study of one thousand forty-three individuals. Spine (Phila Pa 1976). 2009;34 (9):934-940. doi:10.1097/BRS.0b013e3181a01b3f

15. Al-Saeed O, Al-Jarallah K, Raeess M, Sheikh M, Ismail M, Athyal R. Magnetic resonance imaging of the lumbar spine in young arabs with low back pain. Asian Spine J. 2012;6(4):249-256. doi:10.4184/ asj.2012.6.4.249

16. Meucci RD, Fassa AG, Faria NM. Prevalence of chronic low back pain: systematic review. Rev Saude Publica. 2015;49:1. doi:10.1590/ S0034-8910.2015049005874

17. Faur C, Patrascu JM, Haragus H, Anglitoiu B. Correlation between multifidus fatty atrophy and lumbar disc degeneration in low back pain. BMC Musculoskelet Disord. 2019;20(1):414. doi:10.1186/ s12891-019-2786-7
Orthopedic Research and Reviews

\section{Publish your work in this journal}

Orthopedic Research and Reviews is an international, peer-reviewed, open access journal that focusing on the patho-physiology of the musculoskeletal system, trauma, surgery and other corrective interventions to restore mobility and function. Advances in new technologies, materials, techniques and pharmacological agents are particularly

\section{Dovepress}

welcome. The manuscript management system is completely online and includes a very quick and fair peer-review system, which is all easy to use. Visit http://www.dovepress.com/testimonials.php to read real quotes from published authors. 